\title{
Combination of rifaximin and lactulose improves clinical efficacy and mortality in patients with hepatic encephalopathy
}

This article was published in the following Dove Press journal: Drug Design, Development and Therapy

\author{
Zhida Wang' \\ Pei Chu ${ }^{2}$ \\ Wenjin Wang ${ }^{2}$ \\ 'Comprehensive Clinical Skill Training \\ Center, The First Hospital of Lanzhou \\ University, Lanzhou 730030, China: \\ ${ }^{2}$ Department of Emergency, The \\ First Hospital of Lanzhou University, \\ Lanzhou 730030, China
}

Background: Rifaximin and lactulose are common effective agents for hepatic encephalopathy (HE). Whether a combination of rifaximin and lactulose improves the efficacy and mortality in patients with HE compared with lactulose alone needs to be analyzed.

Methods: A systematic search was performed in electronic databases and other sources for possible studies focusing on combination therapy of rifaximin and lactulose for HE between January 2000 and February 2018. A meta-analysis was performed by the method recommended by the Cochrane Collaboration, and estimated effect size was presented as risk difference (RD), 95\% CI, and the number needed to treat (NNT). Subgroup analysis, sensitivity analysis, and Trial Sequence Analysis were comprehensively performed to indicate the source of heterogeneity and risk of bias.

Results: Five randomized and five observational studies involving 2,276 patients were included. Combination therapy had a significant advantage in both clinical efficacy increase (RD 0.26, 95\% CI 0.19-0.32, NNT 5) and mortality decrease (RD $-0.16,95 \%$ CI $-0.20-0.11$, NNT 9) in overall analysis. In the pooled analysis of randomized studies, combination therapy showed similar results in clinical efficacy (RD $0.25,95 \%$ CI $0.16-0.35$, NNT 4) and mortality (RD $-0.22,95 \%$ CI $-0.33-0.12$, NNT 5). Compared with lactulose, hospital stay was also reduced in combination therapy, and there was no significant difference in treatment-related adverse events between the two groups.

Conclusion: Combination of rifaximin and lactulose has beneficial effects on HE. Compared with lactulose alone, additional rifaximin increases clinical efficacy and decreases mortality. However, its effects on different types of HE are still uncertain.

Keywords: rifaximin, lactulose, combination therapy, HE, meta-analysis

\section{Introduction}

Hepatic encephalopathy (HE) is described as a brain dysfunction caused by liver insufficiency and/or portosystemic shunting; it manifests itself as a wide spectrum of neurological or psychiatric abnormalities ranging from subclinical alterations to coma. ${ }^{1}$ $\mathrm{HE}$ is a severe complication of acute or chronic liver failure due to cirrhosis; its prevalence and severity is closely related to the underlying liver status. ${ }^{2}$ The prevalence of minimal $\mathrm{HE}$ in cirrhosis ranged from $20 \%$ to $80 \%$. For overt HE, the prevalence was $10 \%-14 \%$ in general cirrhosis, $16 \%-21 \%$ in decompensated cirrhosis, and $10 \%-50 \%$ in patients who adopted transjugular intrahepatic portosystemic, which was a major minimally invasive shunting surgery. ${ }^{1,3}$ According to an over-5-year analysis, the mortality of HE in hospital was about $15 \% .{ }^{4}$ For cirrhosis patients without stable management or cure, $30 \%-40 \%$ of them suffered repeatedly recurrent HE in their survival periods. ${ }^{5,6}$
Correspondence: Wenjin Wang Department of Emergency, The First Hospital of Lanzhou University, West Donggang Road no. I, Chengguan District, Lanzhou 730030, Gansu Province, China Emailwwjou@I26.com

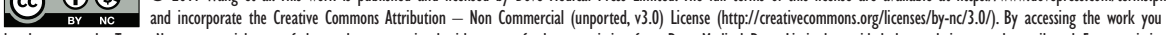
hereby accept the Terms. Non-commercial uses of the work are permitted without any further permission from Dove Medical Press Limited, provided the work is properly atributed. For permision for commercial use of this work, please see paragraphs 4.2 and 5 of our Terms (https://www.dovepress.com/terms.php). 
Besides intensive care and underlying liver disease treatment, $90 \%$ of HE patients can be treated through managing precipitating factors, including gastrointestinal bleeding, excessive protein intake, infection, hypokalemic alkalosis, constipation, hypoxia, or the use of sedatives and tranquilizers. ${ }^{7}$ About $60 \%-80 \%$ of HE patients showed an elevated serum ammonia, and thus therapy was recommended, including non-absorbable disaccharides (NAD), rifaximin, L-ornithine-L-aspartate (LOLA), and branched chain amino acids (BCAA), which mostly aim to reduce the level of serum ammonia. ${ }^{8}$ Among them, lactulose is the first choice and the most widely adopted NAD, while rifaximin emerged as an effective non-absorbed oral antimicrobial agent in recent years. ${ }^{9-11}$ However, the two drugs presented similar clinical effects, which were demonstrated by largescale meta-analysis studies. ${ }^{12}$

Because rifaximin and lactulose had different pharmacological mechanisms for HE, it is important to understand whether a combination of them would further increase the clinical efficacy compared to lactulose alone. Investigated by a series of small-scale studies, ${ }^{13-21}$ the issue was not fully evaluated and has yet to provide a confirmed conclusion. Therefore, we performed a comprehensive systematic review and metaanalysis of published clinical studies aiming to determine the comparative efficacy and safety of combined rifaximin and lactulose with lactulose alone for current HE treatment.

\section{Methods}

\section{Search strategy}

Current meta-analysis was reported in accordance with Preferred Reporting Items for Systematic Reviews and Meta-Analyses Guideline. Comprehensive searches including electronic databases, clinical register centers, scholar search engine and manual search were completed using modified search strategy. Search terms were "hepatic encephalopathy, rifaximin, lactulose, plus, and combination". Online search was carried out by searching the websites of PubMed, EMBASE, the Cochrane Library, CNKI, Wanfang, ClinicalTrials.gov, Google scholar, and Baidu scholar between January 2000 and February 2018. Manual search was done by screening the references and citations of the similar studies. Publication language was limited to English and Chinese.

\section{Study inclusion and data extraction}

Both randomized and observational controlled studies investigating specific topics were considered in our analysis. Relevant meta-analyses and systematic reviews were also searched. Participants were patients suffering from covert (the West Haven criteria, < grade 2) and overt HE (the West Haven criteria, $\geq$ grade 2) due to liver cirrhosis. Combination therapy of rifaximin and lactulose was compared with lactulose alone in the treatment of HE, and the specific dose and usage of rifaximin were not restricted; also, there were no restrictions of the control type (bland control or placebo). Outcome measurements were clinical efficacy, mortality, and treatment-related adverse events. Two reviewers independently assessed the eligibility of all potential citations obtained from initial search, and a third reviewer checked the included studies.

Data extraction was also completed by two independent reviewers. Baseline study information, patients' characteristic, comparison, type of HE, etiology and severity of HE, treatment duration, and follow-up period for evaluation of clinical outcomes were extracted from each study; the number of events of interest in studies were extracted for further statistical analysis purpose. Discrepancies regarding the extraction of data were resolved by the third reviewer.

\section{Outcome measures}

Primary outcome measures included clinical efficacy and mortality. Clinical efficacy was defined as improvement in the HE clinical syndrome with improved neurological status or a significant decrease in the HE index after treatment. Secondary outcome measure was treatment-related adverse events such as severe diarrhea, episodes of intense abdominal pain, and other gastrointestinal system reactions.

\section{Assessment of risk of bias}

Current analysis included both randomized and observational studies, and risk of bias located in each study was independently assessed by two reviewers by using different scales. Modified JADAD scale was used for randomized controlled trials, which contained items assessing sequence generation for the randomization, allocation of treatment concealment, blinding of participant and outcome measures, follow-up, and drop out. Trials with a score of $\leq 3$ (total 7 scores) were considered as having high risk of bias and $>3$ were considered as having low risk of bias. Newcastle-Ottawa Scale (NOS) was used for observational cohort studies, which contained items assessing risk in exposed cohort representativeness, non-exposed cohort selection, ascertainment of exposure, comparability of cohort, interested outcome assessment, and follow-up periods. Studies achieving a score $>4$ (total 9 scores) were considered to be low risk of bias. Specific checklists for randomized controlled trials and observational 
cohort studies of Scottish Intercollegiate Guidelines Network were also used.

\section{Statistical analysis}

Review Manager (version 5.3, Cochrane Center, Copenhagen, Denmark) was used to perform this meta-analysis. In the software, $I^{2}$ and corresponding $P$-value were used to investigate the significance of statistical heterogeneity. Moderate, considerable, and substantial heterogeneity were set based on the value of $I^{2}$ range from $30 \%$ to $60 \%, 60 \%$ to $75 \%$, and $>75 \%$. Clinical heterogeneity was handled by subgroup analysis according to study design (randomized studies or not). According to $I^{2}$ value, random-effects model or fixedeffects model was selected to analyze the data extracted from published reports. For each outcome measure under randomeffects model, the result of fix-effect model was reported only if the difference between the two models existed. Current meta-analysis used risk difference (RD) indicating the effect size of categorical variables and mean difference (MD) indicating the effect size of continuous variables together with their $95 \%$ CI. Both of them represented an average MD between the groups, and $P$-value $<0.05$ was considered to be statistically significant.

For primary outcome measures, the number needed to treat (NNT) was also calculated, which represented the number of patients needed to treat to achieve a different clinical outcome. Based on the data of absolute risk reduction (ARR), experimental event rate (EER), and control event rate (CER) in low risk of bias studies, NNT was obtained as $\mathrm{NNT}=1 / \mathrm{ARR}$ and ARR=CER EER. The $95 \% \mathrm{CI}$ of NNT was calculated based on the lower limit (LL) and upper limit (UL) of $95 \%$ CI of ARR: $\mathrm{NNT}_{\mathrm{LL}}=1 / \mathrm{ARR}_{\mathrm{UL}}$ and $\mathrm{NNT}_{\mathrm{UL}}=1 / \mathrm{ARR}_{\mathrm{LL}}$. Statistical significance was identified by the range of $95 \%$ CI that it did not cross the value of 1 . We further confirmed reliability of the results in Trial Sequential Analyses (TSA) by using TSA software (Copenhagen Trial Unit, Copenhagen, Denmark). The sequential analyses were based on randomeffects model, with $\alpha=5 \%$ and a test power of $80 \%$, and model-based heterogeneity (diversity). The analysis results were judged as highly reliable when the z-curve crossed the trial monitoring curve, which indicated that cumulative result of included trials was relative enough to achieve a certain result even if the required sample size was not reached.

\section{Results}

\section{Study information}

During the search process, 360 citations including 64 duplicates were obtained. After screening the titles and abstracts, 23 studies were assessed for eligibility by reading full-texts (Figure 1). Finally, seven studies with full text and two

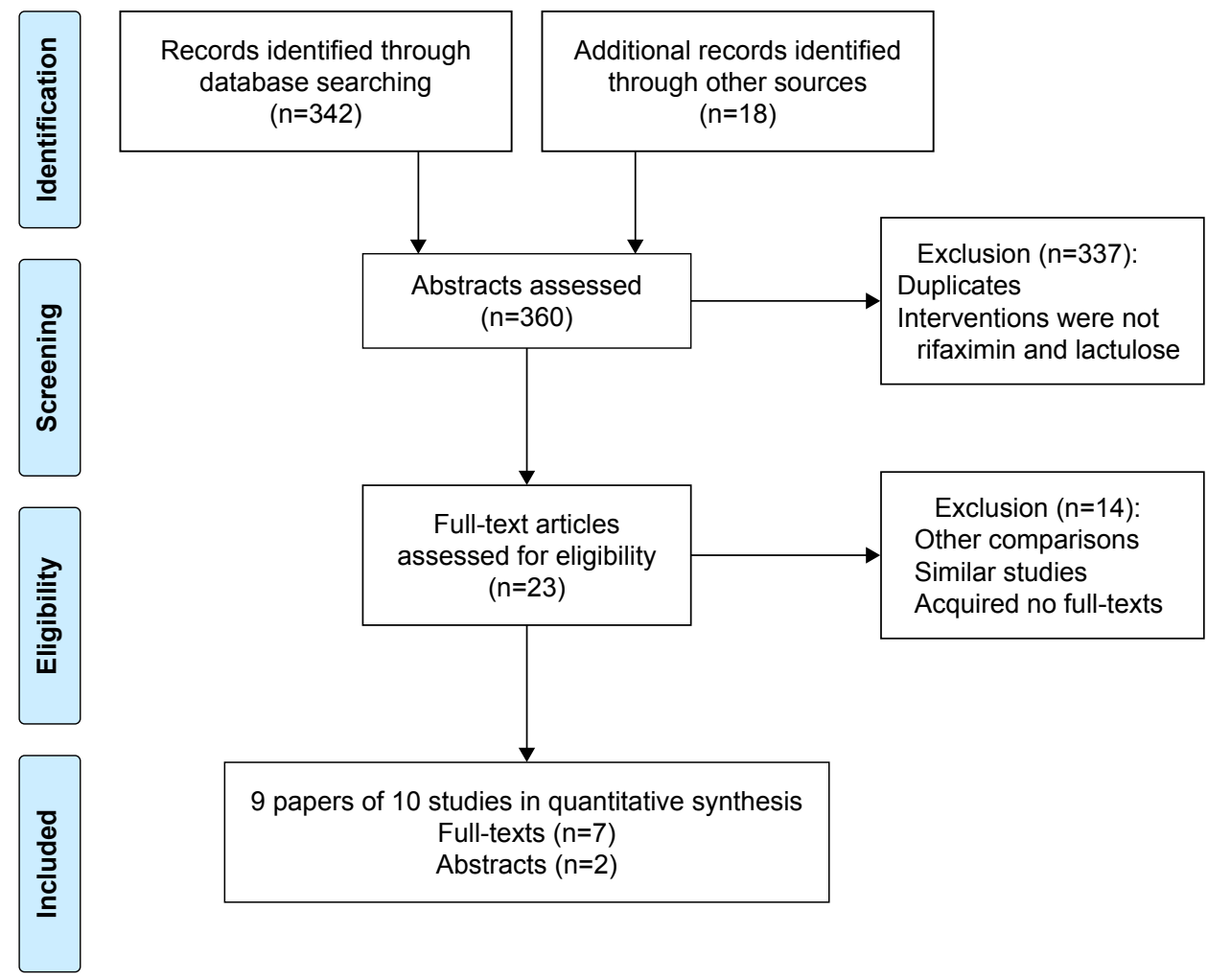

Figure I Process of including studies evaluating combination therapy for hepatic encephalopathy. 


\begin{tabular}{|c|c|c|c|c|c|c|c|c|c|c|}
\hline 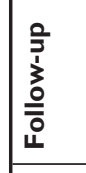 & $\frac{\underline{n}}{\underline{d}}$ & 总 & 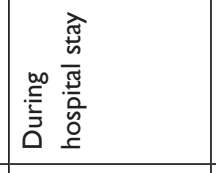 & 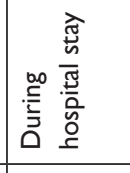 & $\begin{array}{l}\text { 登 } \\
\text { N }\end{array}$ & $\begin{array}{l}\text { 各 } \\
\text { 音 } \\
\underline{\underline{m}}\end{array}$ & 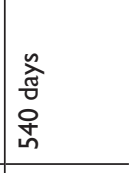 & & 总 & 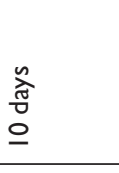 \\
\hline 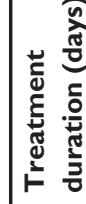 & & 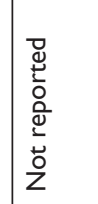 & $\frac{\mathrm{O}}{\mathrm{V}}$ & $\circ$ & $\hat{v}$ & $\frac{\mathrm{O}}{\mathrm{V}}$ & 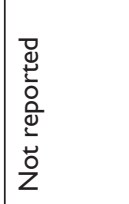 & & $\infty$ & $\frac{\mathrm{O}}{\mathrm{V}}$ \\
\hline | & 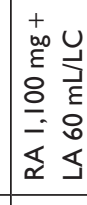 & 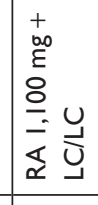 & 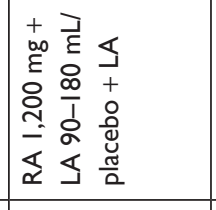 & 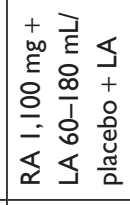 & 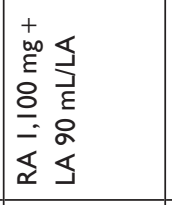 & 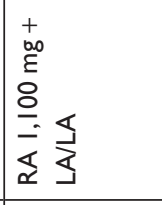 & 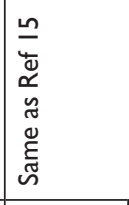 & & 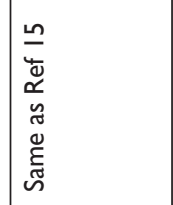 & 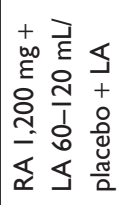 \\
\hline 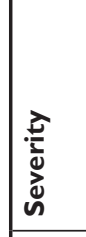 & 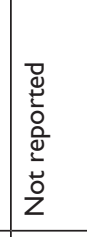 & 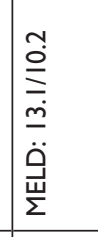 & 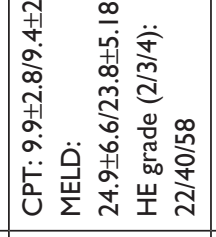 & 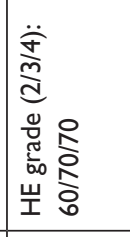 & 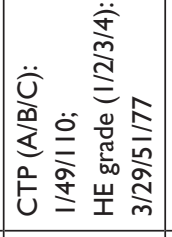 & \begin{tabular}{|l}
$\overline{\bar{\alpha}}$ \\
$\ddot{\underline{\underline{\omega}}}$ \\
$\underline{\underline{\mu}}$ \\
$\underline{\Sigma}$ \\
\end{tabular} & 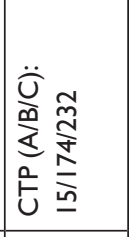 & 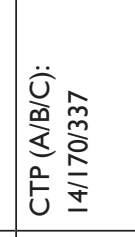 & 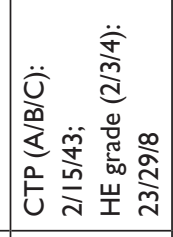 & 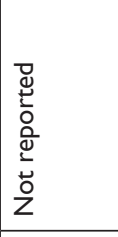 \\
\hline 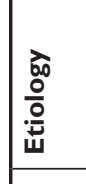 & 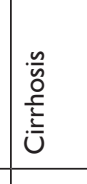 & 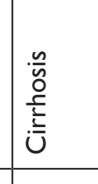 & 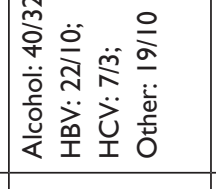 & 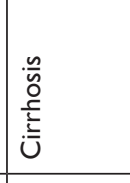 & 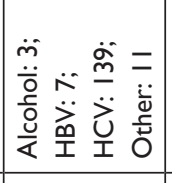 & 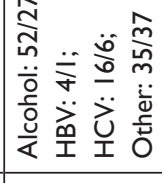 & 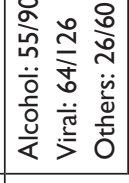 & 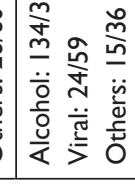 & 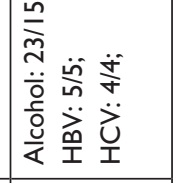 & 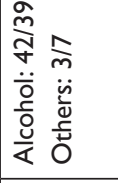 \\
\hline 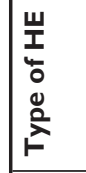 & 뽀 & 포 & 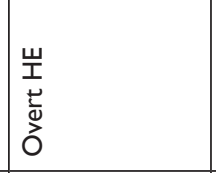 & 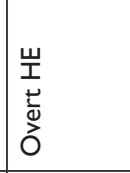 & 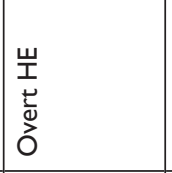 & 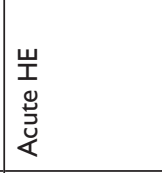 & 崩 & & 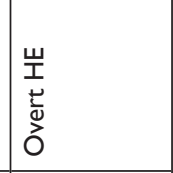 & 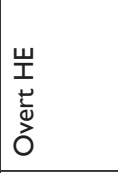 \\
\hline 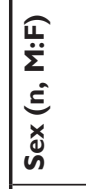 & 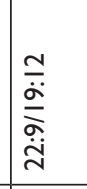 & - & 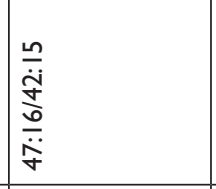 & 商 & 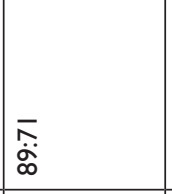 & 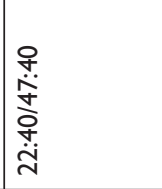 & $\mid$ & 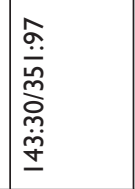 & 芯 & 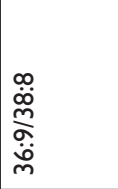 \\
\hline 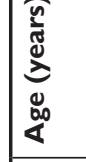 & 总 & - & 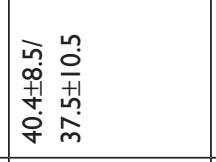 & q & $\begin{array}{l}\infty \\
\vdots \\
\frac{1}{+}\end{array}$ & 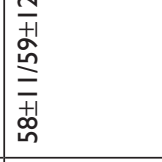 & 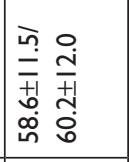 & 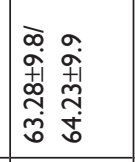 & 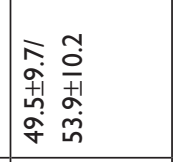 & 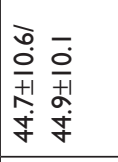 \\
\hline 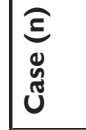 & $\frac{\bar{m}}{\bar{m}}$ & 峛 & $\frac{\hat{n}}{\hat{w}}$ & 음 & 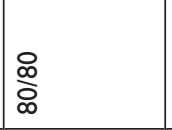 & 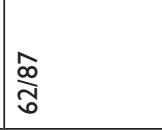 & 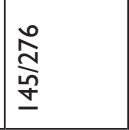 & \begin{tabular}{|l}
$\frac{o}{2}$ \\
$\frac{2}{2}$ \\
$\underline{2}$
\end{tabular} & స్లే & $\begin{array}{l}\frac{9}{3} \\
\frac{3}{3}\end{array}$ \\
\hline 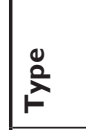 & 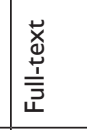 & 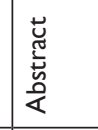 & 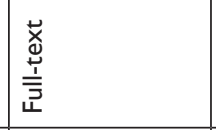 & 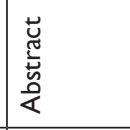 & 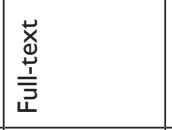 & 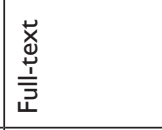 & $\begin{array}{l}\text { 苛 } \\
\frac{\vec{y}}{\vec{z}} \\
\end{array}$ & & 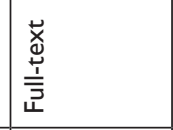 & 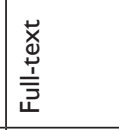 \\
\hline 步 & $\underline{\underline{x}}$ & ¿̆ & $\begin{array}{l}\underline{u} \\
\ddot{x}\end{array}$ & $\underline{\underline{z}}$ & 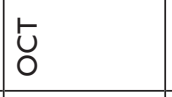 & $\underline{\underline{u}}$ & ö & & Łั & 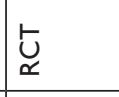 \\
\hline 总 & 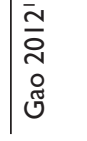 & 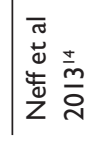 & 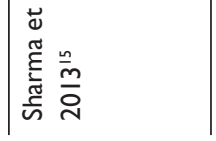 & $\mid$ & 要 & 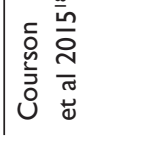 & 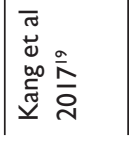 & 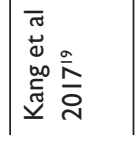 & 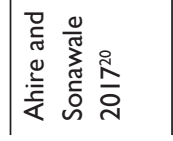 & 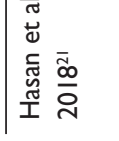 \\
\hline
\end{tabular}


studies with abstracts were included, among them one study divided patients into two groups (with HCC or not) ${ }^{19}$ which were regarded as two separate trials. A total of 771 patients were assigned to combination group (rifaximin and lactulose) and 1,505 patients were assigned to control group (lactulose alone). Demographic characteristics are presented in Table 1. Six studies included overt HE patients (the West Haven criteria, $\geq$ grade 2), ${ }^{15-17,19-21}$ and three studies included new onset and recurrent HE patients without detailed grade of the West Haven criteria. ${ }^{13,14,18}$ The etiology of cirrhosis included alcohol, hepatic virus infection, and others. The severity of HE was judged by the abovementioned HE grade. The Child Pugh score and model for end-stage liver disease score judged the severity of underlying liver disease. Among them, HE grade $>1$ was diagnosed as overt $\mathrm{HE}$, which indicated that clinical findings such as lethargy or apathy, disorientation for time, and obvious personality change were reproducible. The dose of rifaximin was fixed in the studies that $1,100 \mathrm{mg}$ therapy was adopted in five studies, ${ }^{13,14,16-18}$ and $1,200 \mathrm{mg}$ therapy was adopted in four studies. ${ }^{15,19-21}$ The volume of lactulose varied in each study, ranging from $60 \mathrm{~mL}$ to $180 \mathrm{~mL}$ (the amount was $667 \mathrm{mg}$ in $1 \mathrm{~mL}$ ). The treatment duration was mainly less than or equal to 10 days, and only one study reported a maximum treatment duration of 15 days. ${ }^{13}$ The follow-up period for analysis of clinical efficacy and mortality was similar to treatment duration, as the outcomes were mostly measured during hospital stay, except for two studies that had a follow-up period $>180$ days. ${ }^{18,19}$

\section{Risk of bias}

Five randomized studies were assessed by JADAD scale; $;^{13,15,16,18,21}$ three of them had a score $>3^{15,18,21}$ and three of them designed a placebo control. ${ }^{15,16,21}$ Four observational studies were assessed by NOS scale,,$^{14,17,19,20}$ and three of them had a score $>4 .{ }^{17,19,20}$ Both of them were assessed by SIGN scale, where two studies were judged as high quality, ${ }^{19,20}$ five studies were judged as acceptable, and only one study was judged as low quality. ${ }^{14}$ The assessment result of risk of bias is shown in Table 2.

\section{Clinical efficacy}

Six studies evaluated the effects of the combination of rifaximin and lactulose on $\mathrm{HE}^{13,15-17,20,21}$ (Figure 2). The metaanalysis result showed that combination therapy significantly increased clinical efficacy compared with lactulose alone in HE patients (RD 0.19, 95\% CI 0.09-0.29, $P=0.0002$ ). The heterogeneity value of $I^{2}$ was $59 \%$, and one study was identified to be responsible for this moderate heterogeneity
Table 2 Risk of bias assessment of the included studies

\begin{tabular}{l|l|l|l}
\hline Studies & SIGN & JADAD & NOS \\
\hline Gao $2012^{13}$ & Acceptable & 3 & - \\
Neff et al $2013^{14}$ & Low quality & - & 4 \\
Sharma et al $2013^{15}$ & High quality & 6 & - \\
Gill et al $2014^{16}$ & Acceptable & 3 & - \\
Miu et al 2014 & Acceptable & - & 5 \\
Courson et al $2015^{18}$ & Acceptable & 4 & - \\
Kang et al $2017^{19}$ & High quality & - & 9 \\
Ahire and Sonawale $2017^{20}$ & High quality & - & 8 \\
Hasan et al $2018^{21}$ & High quality & 6 & - \\
\hline
\end{tabular}

Abbreviations: JADAD, JADAD scale; NOS, Newcastle-Ottawa Scale; SIGN, Scottish Intercollegiate Guidelines Network.

through inverted funnel plot. ${ }^{21}$ After excluding it, the value of $I^{2}$ was reduced to $35 \%$; the sensitivity analysis result was consistent with that of before (RD $0.26,95 \%$ CI $0.19-0.32$, $P<0.00001)$.

Randomized studies were regarded to be better than non-randomized studies in the study design; accordingly, we analyzed the result based on only randomized studies (Figure 3). In the new pooled analysis, three studies containing $342 \mathrm{HE}$ patients also demonstrated a significant increase in clinical efficacy of combination therapy (RD 0.25, 95\% CI $\left.0.16-0.35, P<0.00001, I^{2}=0 \%\right){ }^{13,15,16}$

The NNT was 5 (95\% CI 3.45-11.11) in primary analysis, while it was 4 in sensitivity analysis (95\% CI 3.13-5.26) and randomized study analysis ( $95 \%$ CI $2.86-6.25$ ). In TSA analysis, we set $\alpha$ to $5 \%$, test power to $80 \%$, control group incidence to $54 \%$, relative risk reduction to $-26 \%$, and heterogeneity correction to $35 \%$. The required sample size was 615 participants. The monitoring boundary was crossed in 2013, which confirmed the reliability of current meta-analysis result with enough required sample size (Figure 4). Inverted funnel plot indicated a low risk of bias (Figure 5).

\section{Mortality}

Seven studies reported the data of mortality ${ }^{15-21}$ (Figure 6). In the pooled analysis, combination therapy significantly reduced the mortality in HE patients compared with lactulose alone (RD $-0.11,95 \% \mathrm{CI}-0.19$ to $-0.03, P=0.009$ ). Considerable heterogeneity was found $\left(I^{2}=70 \%\right)$. Two studies were identified to be different with others through an inverted funnel plot. ${ }^{18,21}$ Exclusion of the studies decreased the value of $I^{2}$ to $41 \%$, and sensitivity analysis result showed a similar trend to before (RD $-0.16,95 \% \mathrm{CI}-0.20$ to -0.11 , $P<0.00001)$.

In the new pooled analysis based on randomized studies, two studies containing 280 patients demonstrated a significant 


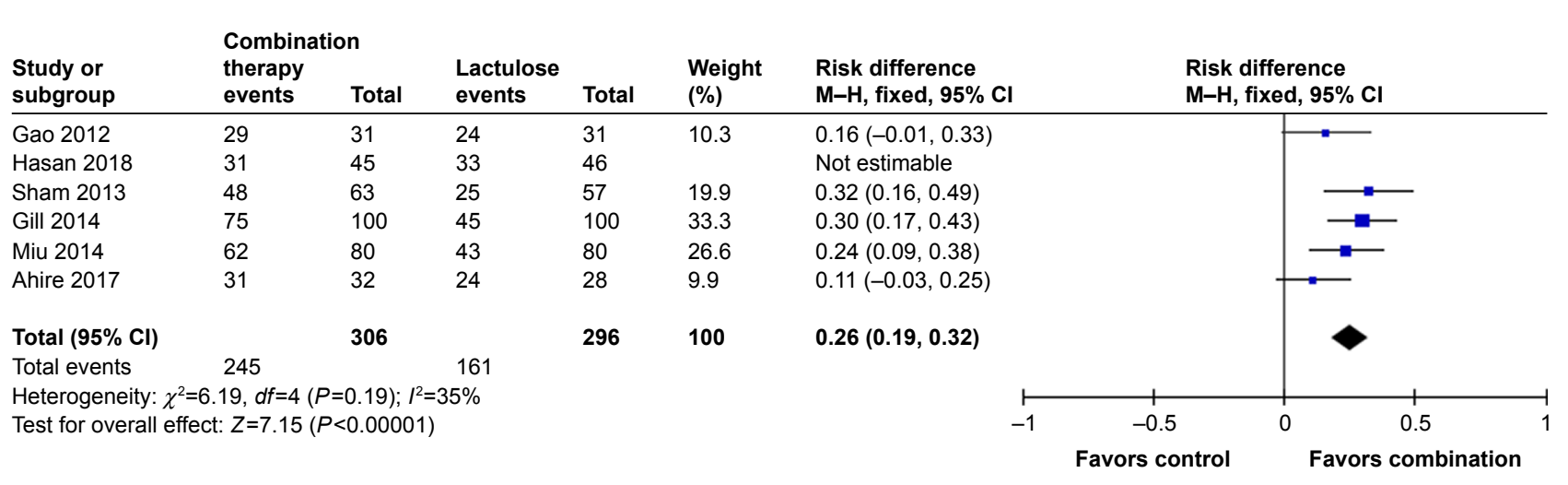

Figure 2 Meta-analysis result of clinical efficacy in overall analysis between combination therapy and lactulose alone. Abbreviation: $\mathrm{M}-\mathrm{H}$, Mantel-Haenszel test.

decrease of mortality in combination therapy $(\mathrm{RD}-0.22,95 \%$ CI -0.33 to $-0.12, P<0.0001, I^{2}=0 \%$; Figure 7$){ }^{15,16}$

The NNT was $9(95 \%$ CI 5.26-33.33) in primary analysis, 6 (95\% CI 5.00-9.09) in the sensitivity analysis, and 5 (95\% CI 3.03-8.33) in randomized study analysis. In TSA analysis, we set $\alpha$ to $5 \%$, power to $80 \%$, control group incidence to $67 \%$, relative risk reduction to $16 \%$, and heterogeneity correction to $41 \%$. The required sample size was 1,248 participants. The monitoring boundary was crossed in 2014, which confirmed the reliability of current meta-analysis result with enough sample size (Figure 8). Inverted funnel plot indicated low risk of bias (Figure 9).

Based on available data of one study including 120 patients (HE grade 2/3/4 was 22/40/58) with a mortality of $35.83 \% ;{ }^{15}$ deaths were caused by sepsis $(7 / 17, P=0.01)$, hepatorenal syndrome $(4 / 7, P>0.05)$, and gastrointestinal bleeding $(4 / 4, P>0.05)$ in each group.

\section{Hospital stay}

Three studies reported the data of hospital stay. Two of them were pooled analyzed, and meta-analysis results showed that combination therapy significantly reduced the duration of hospital stay (MD $-2.89,95 \% \mathrm{CI}-3.52$ to -2.25 , $P<0.00001) .^{15,16}$ The other study only reported the data of median hospital stay between the groups $(6 / 8, P=0.09),{ }^{18}$ which found no significant difference.

\section{Adverse events}

Four studies compared the treatment-related adverse events (Figure 10). In the pooled analysis, there was no significant difference in combination therapy and lactulose alone (RD $-0.06,95 \% \mathrm{CI}-0.24$ to $0.13, P=0.56$ ). One study was found to be responsible for the substantial heterogeneity ( $I^{2}=90 \%$ ); after excluding it, ${ }^{14}$ the $I^{2}$ value was obviously reduced $\left(I^{2}=0 \%\right)$. The sensitivity result was similar to before (RD $0.0,95 \% \mathrm{CI}-0.03$ to $0.02, P=0.63$ ).

\section{Discussion}

$\mathrm{HE}$ is one of the most common causes of death and would cause many precipitating factors in cirrhosis patients. ${ }^{22}$ According to the recommendations in the 2014 practice guideline, controlling precipitating factors is of paramount importance in the management of HE. Besides precipitating factor management, lactulose is the first recommended agent. Rifaximin, oral BCAA, intravenous LOLA, neomycin, and metronidazole can also be used as alternative or additional agents. ${ }^{1}$ However, high-level evidence such as meta-analysis or systematic review is still lacking.

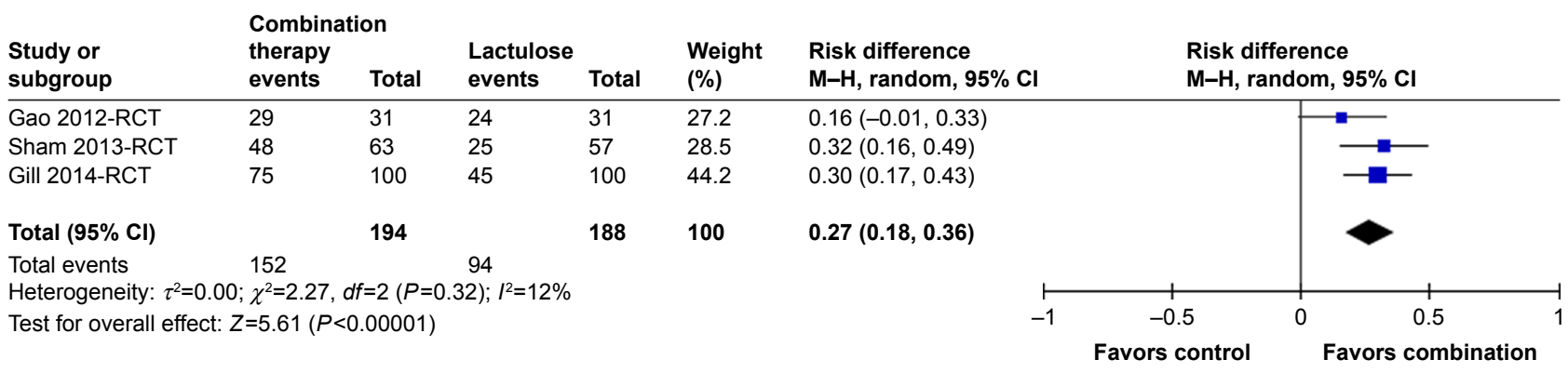

Figure 3 Meta-analysis result of clinical efficacy in randomized controlled trials between combination therapy and lactulose alone. Abbreviation: RCT, randomized controlled trial. 


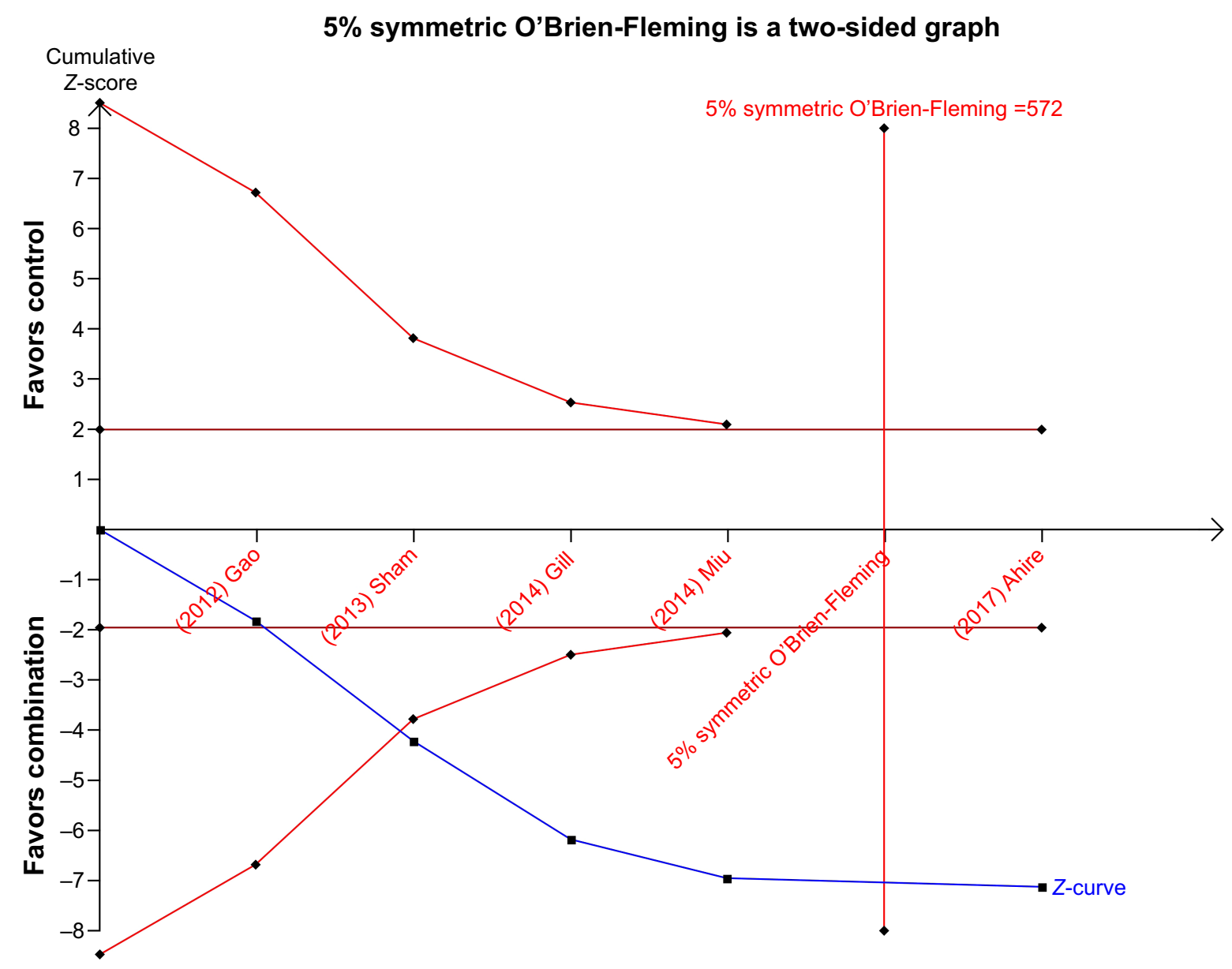

Figure 4 Trial sequence analysis result of clinical efficacy.

The current meta-analysis was the first to quantitatively evaluate the effective effects of combination therapy of rifaximin and lactulose vs lactulose alone for the management of HE. We included five randomized and five observational controlled studies, with a sample size of 2,276 HE patients, and demonstrated that combination therapy

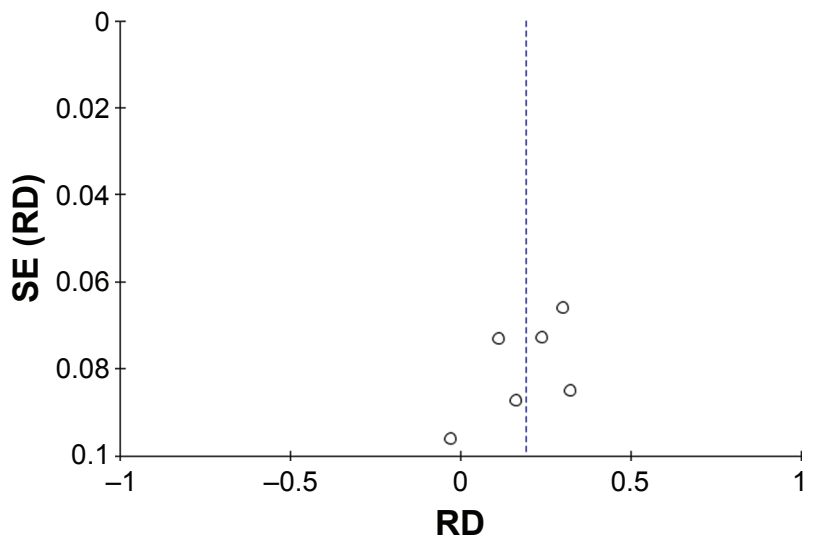

Figure 5 Inverted funnel plot of clinical efficacy. Abbreviations: RD, risk difference; SE, standard error. significantly improved the clinical efficacy, mortality, and hospital stay without increasing treatment-related adverse events in HE.

As stated within the practice guideline, current definition, diagnosis, classification, or the treatment of HE are not universally accepted, and the complex pathogenesis of HE is difficult to clarify. ${ }^{1}$ Currently, clinical observations always exhibit a high average level of ammonia in high grade HE patients; thus, the ammonia hypothesis is a widely accepted premise that leads to frequent assessment of ammonia concentrations. ${ }^{23}$ Conventional pharmacologic treatment of HE consisted of NAD since 1966, which mainly included lactulose and lactitol. ${ }^{9}$ A meta-analysis including 38 randomized trials published in 2016 had sufficiently confirmed the effectiveness of the routine use of NAD to reduce the production and absorption of gut-derived neurotoxin ammonia in clinical practice, as well as the potential roles of catharsis, ammonia metabolism, and gut microbiome adjustment. ${ }^{9,24}$

For a specific HE patient, the serum level of ammonia sometimes did not correlate with the severity of clinical symptoms. 


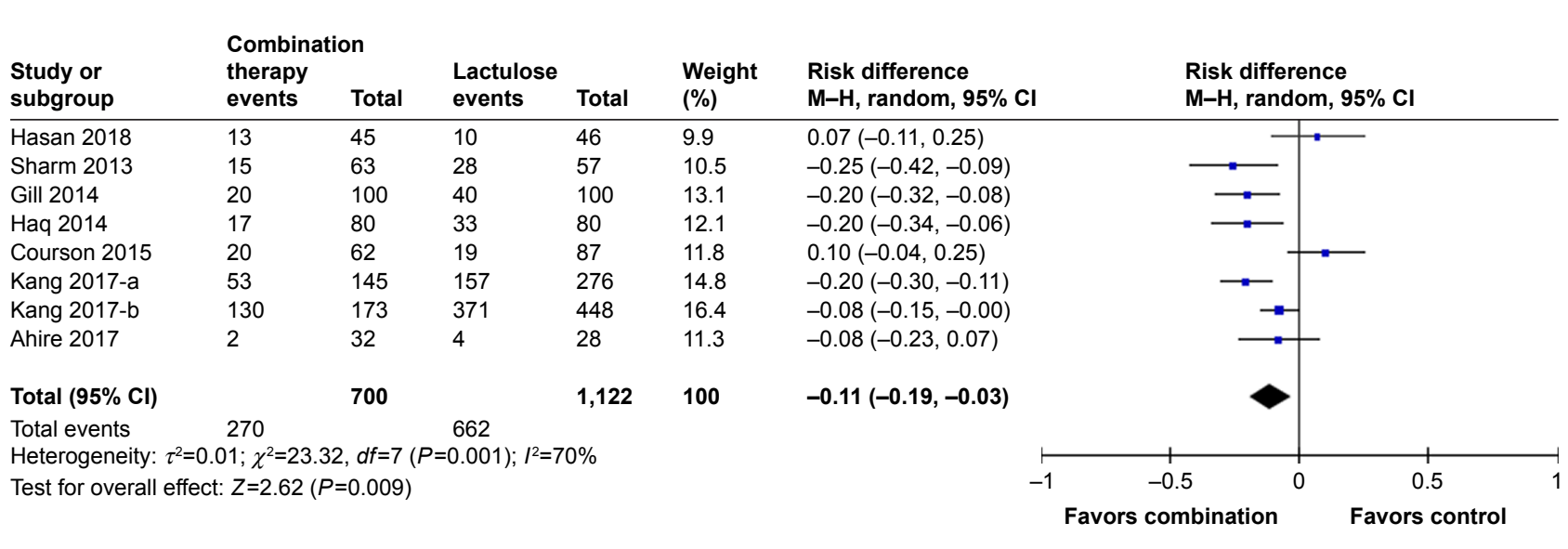

Figure 6 Meta-analysis result of mortality in overall analysis between combination therapy and lactulose alone.

Meanwhile, clinical analysis reported that lactulose therapy was related to a non-response rate as high as $22 \% .{ }^{25}$ Accordingly, improved therapy based on lactulose is warranted since no certain method was introduced to identify a non-responder of lactulose therapy. Confirmed by sensitivity analysis, subgroup analysis, Trial Sequential Analysis, and publication bias analysis, our meta-analysis shows that a combination of rifaximin to lactulose can significantly improve both clinical efficacy of HE manifestations (NNT =4) and mortality $(\mathrm{NNT}=5)$ mainly for overt HE patients. Based on limited data in one randomized study, ${ }^{15}$ the cause of deaths was significantly reduced only in aspect of sepsis, which indicated that combination therapy may improve clinical outcome by reducing the incidence of severe and systemic infection and inflammation. In past years, multiple factors were included in the explanation of HE progress; among them systemic inflammation and infection burden were originated and induced by gut because of bacterial overgrowth and gut-derived endotoxin seemed to be the most attractive explanation. ${ }^{19,26,27}$ Therapies targeting the infection and inflammation deriving from the gut were effective, and a new development of non-absorbed rifaximin with patient tolerance became a promising agent. ${ }^{11}$ The therapeutic actions of rifaximin were reported to be twofold in reducing the number of ammonia-producing enteric bacteria in the gastrointestinal tract, and would initially inhibit the occurrence of systemic inflammation because of enteric bacteria translocationinduced endotoxin. ${ }^{26}$ Therefore, improved clinical outcomes in HE patients after applying additional rifaximin may be achieved by interrupting the pathway of gut-derived local and systematic infection and inflammations, as well as by inhibiting the potential of involvement and injury in organs including liver, lung, brain, and the body's immune system. All of these would contribute to a significant reduction in length of hospital stay.

Lactulose is reported to be associated with increased mild adverse events in the gastrointestinal system, such as diarrhea and abdominal pain. ${ }^{28}$ Although it may lead to some temporary discomfort in HE patients, most do not need clinical interventions. Compared with lactulose, rifaximin can be better tolerated with fewer incidences of adverse events. It was reported that compared with other absorbed and systemic antibiotics, non-absorbed rifaximin induced lower risk of bacteria resistance; its plasma concentration was negligible, which indicated a very low risk of bacteria selection outside the gut. ${ }^{15,29}$ In the current study, a combination of the two agents did not increase any risk of adverse events related to the treatment.

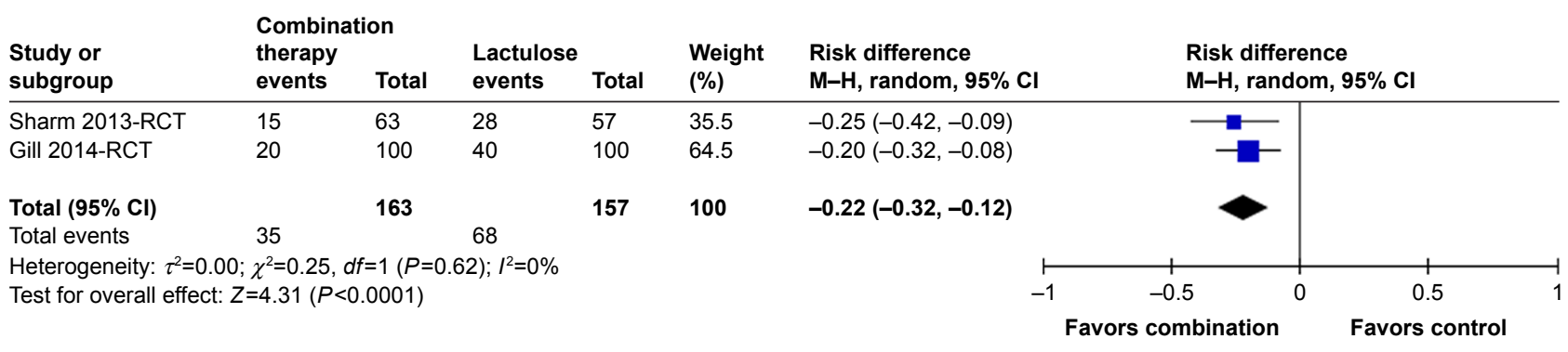

Figure 7 Meta-analysis result of mortality in randomized controlled trial between combination therapy and lactulose alone. 


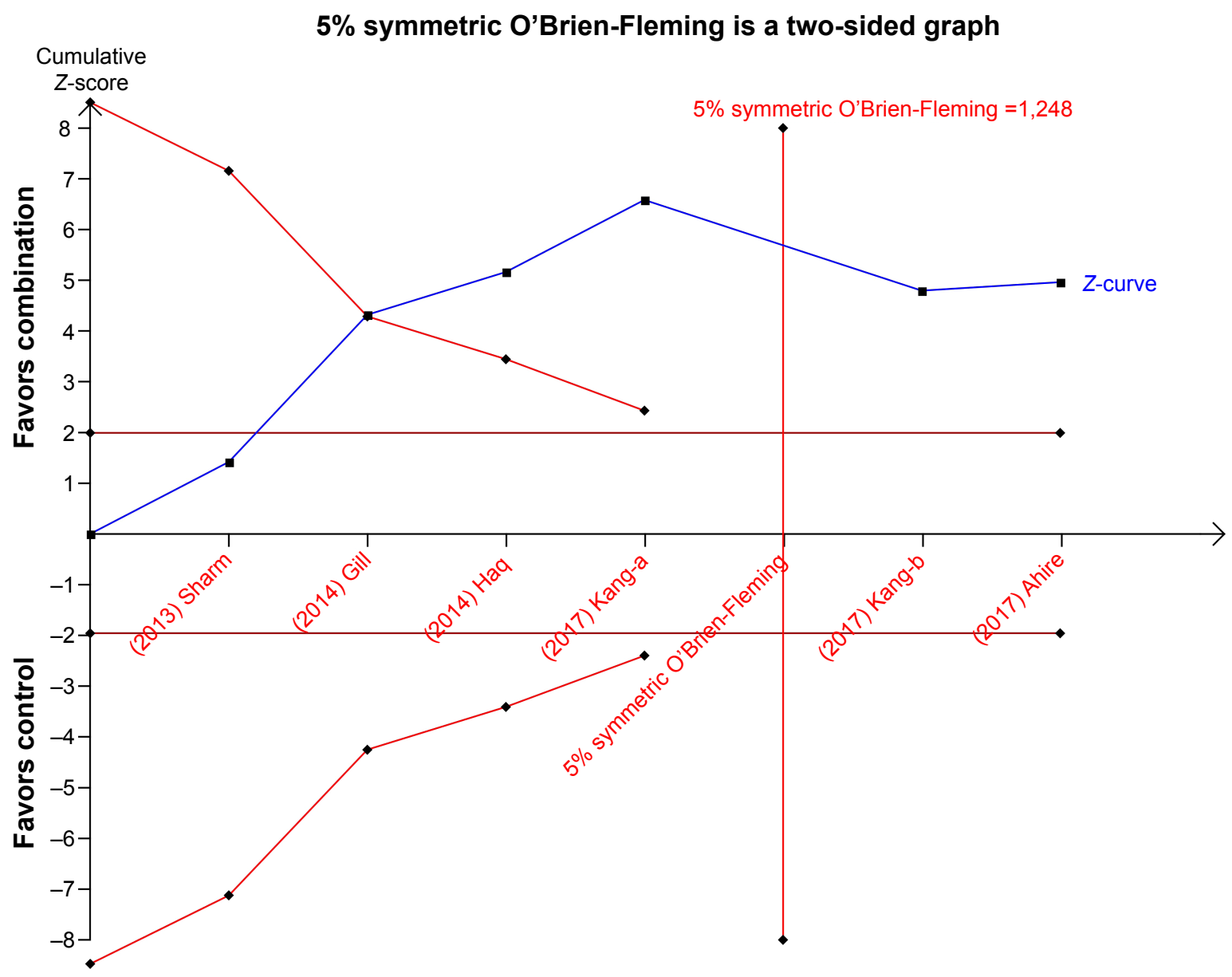

Figure 8 Trial sequence analysis result of mortality.

Limitations existing in the meta-analysis mainly included study quality, outcome measures, and targeted population. Randomized studies are regarded as higher level evidence than non-randomized studies. The current meta-analysis included both randomized and non-randomized studies to enable a large enough sample size. For the sensitivity

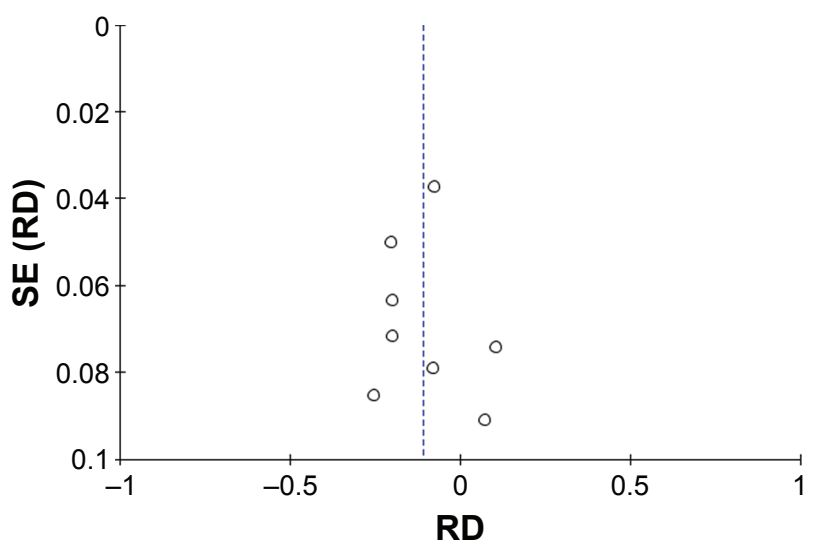

Figure 9 Inverted funnel plot of mortality. Abbreviation: RD, risk difference. analysis, excluding non-randomized studies did not alter the results despite a small difference in the value of effect size. For outcome measures, the current meta-analysis can only analyze data extracted from original reports. Clinical efficacy was judged by different centers; this difference across the studies may induce moderate heterogeneity although it is comparable between groups within each study. Development of a valid and standard test scale of HE is necessary in future research. Currently, HE treatment is mainly targeting at overt HE, because of lower diagnosis rate in covert HE. Combination therapy achieves better clinical outcomes than lactulose alone. ${ }^{8,30}$ However, whether rifaximin and lactulose therapy should be immediately recommended as the first-line treatment for overt HE or an additive therapy of rifaximin for only lactulose-non-response overt HE still needs to be determined. Kang et al performed a cost-analysis study, and showed that the 1-year incremental cost was as high as $\$ 85,560$ to increase the survival rate in one patient. ${ }^{19}$ In another study, Courson et al supposed that although the cost of rifaximin was high, it reduced the overall cost in 


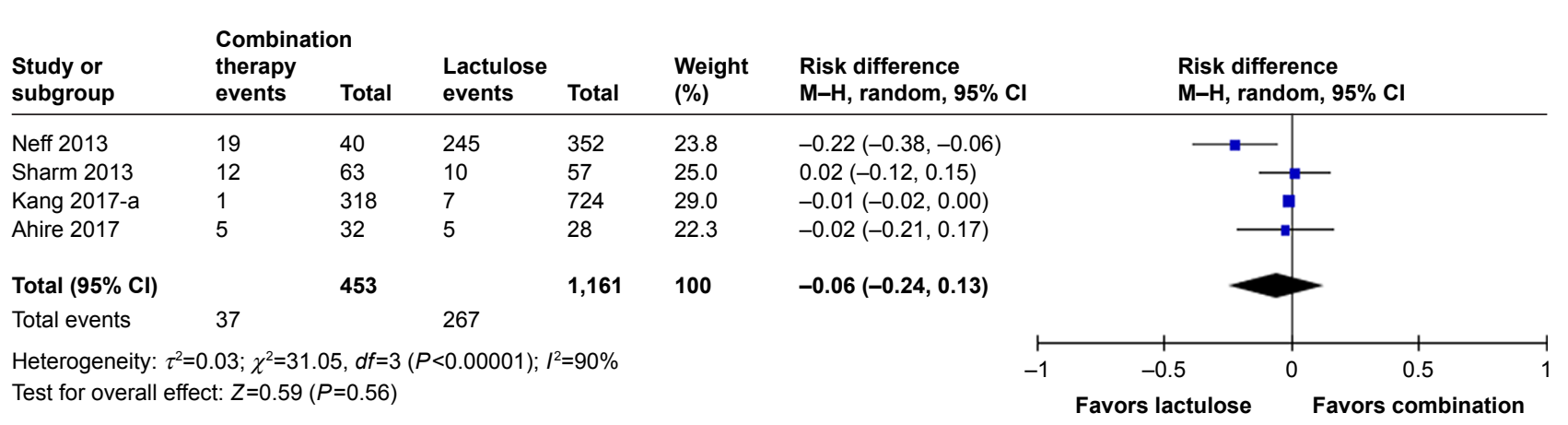

Figure 10 Meta-analysis result of adverse event in overall analysis between combination therapy and lactulose alone.

patients receiving combination therapy than lactulose alone. ${ }^{18}$ Further cost-effective analyses are needed.

Combination therapy of rifaximin and lactulose has beneficial effects on HE. Compared with lactulose alone, combination therapy increases clinical efficacy and decreases mortality in HE patients. However, its effects on different types of HE are still uncertain.

\section{Conclusion}

Combination therapy of rifaximin and lactulose has beneficial effects on HE. Compared with lactulose alone, combination therapy increases clinical efficacy and decreases mortality in HE patients. However, its effects on different types of HE is still uncertain.

\section{Disclosure}

The authors report no conflicts of interest in this work.

\section{References}

1. Vilstrup H, Amodio P, Bajaj J, et al. Hepatic encephalopathy in chronic liver disease: 2014 Practice Guideline by the American Association for the Study of Liver Diseases and the European Association for the Study of the Liver. Hepatology. 2014;60(2):715-735.

2. Riggio O, Ridola L, Pasquale C, et al. Evidence of persistent cognitive impairment after resolution of overt hepatic encephalopathy. Clin Gastroenterol Hepatol. 2011;9(2):181-183.

3. Pereira K, Carrion AF, Salsamendi J, Doshi M, Baker R, Kably I. Endovascular Management of Refractory Hepatic Encephalopathy Complication of Transjugular Intrahepatic Portosystemic Shunt (TIPS): Comprehensive Review and Clinical Practice Algorithm. Cardiovasc Intervent Radiol. 2016;39(2):170-182.

4. Stepanova M, Mishra A, Venkatesan C, Younossi ZM. In-hospital mortality and economic burden associated with hepatic encephalopathy in the United States from 2005 to 2009. Clin Gastroenterol Hepatol. 2012;10(9):1034.e1-1041.e1.

5. Amodio P, Del Piccolo F, Pettenò E, et al. Prevalence and prognostic value of quantified electroencephalogram (EEG) alterations in cirrhotic patients. J Hepatol. 2001;35(1):37-45.

6. Cordoba J, Ventura-Cots M, Simón-Talero M, et al; CANONIC Study Investigators of EASL-CLIF Consortium. Characteristics, risk factors, and mortality of cirrhotic patients hospitalized for hepatic encephalopathy with and without acute-on-chronic liver failure (ACLF). J Hepatol. 2014;60(2):275-281.
7. Jiang Q, Jiang XH, Zheng MH, Jiang LM, Chen YP, Wang L. Rifaximin versus nonabsorbable disaccharides in the management of hepatic encephalopathy: a meta-analysis. Eur J Gastroenterol Hepatol. 2008;20(11):1064-1070.

8. Zhu GQ, Shi KQ, Huang S, et al. Systematic review with network metaanalysis: the comparative effectiveness and safety of interventions in patients with overt hepatic encephalopathy. Aliment Pharmacol Ther. 2015;41(7):624-635.

9. Gluud LL, Vilstrup H, Morgan MY. Nonabsorbable disaccharides for hepatic encephalopathy: A systematic review and meta-analysis. Hepatology. 2016;64(3):908-922.

10. Kimer N, Krag A, Møller S, Bendtsen F, Gluud LL. Systematic review with meta-analysis: the effects of rifaximin in hepatic encephalopathy. Aliment Pharmacol Ther. 2014;40(2):123-132.

11. Bass NM, Mullen KD, Sanyal A, et al. Rifaximin treatment in hepatic encephalopathy. N Engl J Med. 2010;362(12):1071-1081.

12. Wu D, Wu SM, Lu J, Zhou YQ, Xu L, Guo CY. Rifaximin versus Nonabsorbable Disaccharides for the Treatment of Hepatic Encephalopathy: A Meta-Analysis. Gastroenterol Res Pract. 2013;2013:236963.

13. Gao ZM. Clinical observation of rifaximin combined with lactulose for the treatment of hepatic encephalopathy[J]. J China Tradit Chinese Med Inform. 2012;4(2):381.

14. Neff GW, Flamm SL, Mullen KD, et al. Su1298 Improved Outcomes in Hepatic Encephalopathy Using Rifaximin Monotherapy Compared to Rifaximin and Lactulose Combination Therapy. Gastroenterology. 2013;144(5):S451.

15. Sharma BC, Sharma P, Lunia MK, Srivastava S, Goyal R, Sarin SK. A randomized, double-blind, controlled trial comparing rifaximin plus lactulose with lactulose alone in treatment of overt hepatic encephalopathy. Am J Gastroenterol. 2013;108(9):1458-1463.

16. Gill ML, Niaz T, Aziz H, Khan S. P440 outcomes of rifaximin plus lactulose versus lactulose alone in treatment of overt hepatic encephalopathy. J Hepatol. 2014;60(1):S215-S215.

17. Miu H, Salim A, Afzal MS, Malik K, Amin J, Butt AK. Comparison of Rifaximin and Lactulose With Lactulose Alone in the Treatment of Acute Hepatic Encephalopathy in Patients With Liver Cirrhosis[J]. Proceeding S.Z.P.G.M.I. 2014;28(2):115-119.

18. Courson A, Jones GM, Twilla JD. Treatment of Acute Hepatic Encephalopathy: Comparing the Effects of Adding Rifaximin to Lactulose on Patient Outcomes. J Pharm Pract. 2016;29(3):212-217.

19. Kang SH, Lee YB, Lee JH, et al. Rifaximin treatment is associated with reduced risk of cirrhotic complications and prolonged overall survival in patients experiencing hepatic encephalopathy. Aliment Pharmacol Ther. 2017;46(9):845-855.

20. Ahire K, Sonawale A. Comparison of Rifaximin Plus Lactulose with the Lactulose Alone for the Treatment of Hepatic Encephalopathy. J Assoc Physicians India. 2017;65(8):42.

21. Hasan S, Datta S, Bhattacherjee S, et al. A Randomized Controlled Trial Comparing the Efficacy of a Combination of Rifaximin and Lactulose with Lactulose only in the Treatment of Overt Hepatic Encephalopathy[J]. J Assoc Phys India. 2018;66:32. 
22. Tapper EB, Jiang ZG, Patwardhan VR. Refining the ammonia hypothesis: a physiology-driven approach to the treatment of hepatic encephalopathy. Mayo Clin Proc. 2015;90(5):646-658.

23. Qureshi MO, Khokhar N, Shafqat F. Ammonia levels and the severity of hepatic encephalopathy. J Coll Physicians Surg Pak. 2014; 24(3):160-163.

24. Patel VC, White H, Støy S, Bajaj JS, Shawcross DL. Clinical science workshop: targeting the gut-liver-brain axis. Metab Brain Dis. 2016; 31(6):1327-1337.

25. Sharma P, Sharma BC, Sarin SK. Predictors of nonresponse to lactulose in patients with cirrhosis and hepatic encephalopathy. Eur $J$ Gastroenterol Hepatol. 2010;22(5):526-531.

26. Tranah TH, Vijay GK, Ryan JM, Shawcross DL. Systemic inflammation and ammonia in hepatic encephalopathy. Metab Brain Dis. 2013; 28(1):1-5
27. Giannelli V, Di Gregorio V, Iebba V, et al. Microbiota and the gut-liver axis: bacterial translocation, inflammation and infection in cirrhosis. World J Gastroenterol. 2014;20(45):16795-16810.

28. Luo M, Li L, Lu CZ, Cao WK. Clinical efficacy and safety of lactulose for minimal hepatic encephalopathy: a meta-analysis. Eur J Gastroenterol Hepatol. 2011;23(12):1250-1257.

29. Yang J, Lee HR, Low K, Chatterjee S, Pimentel M. Rifaximin versus other antibiotics in the primary treatment and retreatment of bacterial overgrowth in IBS. Dig Dis Sci. 2008;53(1):169-174.

30. Gluud LL, Dam G, Borre M, et al. Lactulose, rifaximin or branched chain amino acids for hepatic encephalopathy: what is the evidence? Metab Brain Dis. 2013;28(2):221-225.

\section{Publish your work in this journal}

Drug Design, Development and Therapy is an international, peerreviewed open-access journal that spans the spectrum of drug design and development through to clinical applications. Clinical outcomes, patient safety, and programs for the development and effective, safe, and sustained use of medicines are the features of the journal, which has also been accepted for indexing on PubMed Central. The manuscript management system is completely online and includes a very quick and fair peer-review system, which is all easy to use. Visit http://www.dovepress.com/testimonials.php to read real quotes from published authors.

Submit your manuscript here: http://www.dovepress.com/drug-design-development-and-therapy-journal 